\title{
Double-layer Bose-Einstein condensates: A quantum phase transition in the transverse direction, and reduction to two dimensions
}

\author{
Mateus C. P. dos Santos, ${ }^{1}$ Boris A. Malomed, ${ }^{2,3}$ and Wesley B. Cardoso ${ }^{1, *}$ \\ ${ }^{1}$ Instituto de Física, Universidade Federal de Goiás 74.690-970, Goiânia, Goiás, Brazil \\ ${ }^{2}$ Department of Physical Electronics, School of Electrical Engineering, Faculty of Engineering, \\ Tel Aviv University, and Center for Light-Matter Interaction, Tel Aviv University, Tel Aviv 69978, Israel \\ ${ }^{3}$ Instituto de Alta Investigación, Universidad de Tarapacá, Casilla 7D, Arica, Chile
}

\begin{abstract}
We revisit the problem of the reduction of the three-dimensional (3D) dynamics of Bose-Einstein condensates, under the action of strong confinement in one direction ( $z$ ), to a 2D mean-field equation. We address this problem for the confining potential with a singular term, viz., $V_{z}(z)=2 z^{2}+\zeta^{2} / z^{2}$, with constant $\zeta$. A quantum phase transition is induced by the latter term, between the ground state (GS) of the harmonic oscillator and the 3D condensate split in two parallel non-interacting layers, which is a manifestation of the "superselection" effect. A realization of the respective physical setting is proposed, making use of resonant coupling to an optical field, with the resonance detuning modulated along $z$. The reduction of the full 3D Gross-Pitaevskii equation (GPE) to the 2D nonpolynomial Schrödinger equation (NPSE) is based on the factorized ansatz, with the $z$-dependent multiplier represented by an exact GS solution of the 1D Schrödinger equation with potential $V(z)$. For both repulsive and attractive signs of the nonlinearity, the 2D NPSE produces GS and vortex states, that are virtually indistinguishable from the respective numerical solutions provided by full 3D GPE. In the case of the self-attraction, the threshold for the onset of the collapse, predicted by the 2D NPSE, is also virtually identical to its counterpart obtained from the 3D equation. In the same case, stability and instability of vortices with topological charge $S=1,2$, and 3 are considered in detail. Thus, the procedure of the spatial-dimension reduction, $3 \mathrm{D} \rightarrow 2 \mathrm{D}$, produces very accurate results, and it may be used in other settings.
\end{abstract}

\section{INTRODUCTION}

Bose-Einstein condensates (BECs) have become a versatile platform for realization of various phenomena, such as the production of bright [1-4] and dark [7] solitons, dark-bright complexes [8], vortices [9] and vortex-antivortex dipoles [10-13], persistent flows in the toroidal geometry [14-16], skyrmions [17], emulation of gauge fields [18] and spin-orbit coupling [19], quantum Newton's cradles [20], Anderson localization of matter waves [21, 22], rogue waves [23], quantum droplets (self-trapped states supported by beyond-mean-field interactions) [24-33], etc. Further details can be found in reviews, both earlier [34-44] and more recent ones [4553].

Lower-dimensional BECs, i.e., bosonic gases tightly confined in one or two transverse direction by a strong potential, make it possible to study specific phase transitions and collective excitations in quantum settings $[54,55]$. In particular, studies of quasi-two-dimensional (quasi-2D) BEC with embedded 2D potentials have drawn much interest $[36,56]$. In this connection, approximations which make it possible to reduce the underlying 3D Gross-Pitaevskii equation (GPE) to effective $1 \mathrm{D}[57-71]$ and $2 \mathrm{D}[63,64,66,67,73-78]$ equations have been elaborated. In particular, effective lowdimensional equations were developed in Refs. [72], [73], and [74]. In the former work, the adiabatic ap-

\footnotetext{
*wesleybcardoso@ufg.br
}

proximation was applied, using an appropriate analytical expression for the local chemical potential, to eliminate the transverse dimensions and derive an effective $1 \mathrm{D}$ equation that governs the axial mean-field dynamics of a strongly elongated (cigar-shaped) BEC with repulsive interatomic interactions. On the other hand, in Ref. [57] effective 1D and 2D time-dependent nonpolynomial nonlinear Schrödinger equations (NPSEs) were derived with the help of the variational approximation, which accounts for the structure of the condensate in the transverse directions. The use of the effective equations with lower dimensionality is quite relevant, as such simplified models may help one to gain deeper insight into the underlying dynamics, as well as to reduce the cost of the computational work. In the experiment, lowdimensional BEC can be created by means of the same technique which is used in the 3D setting, i.e., laser cooling of atoms in magnetic and/or optical trapping potentials $[27,79]$.

In this work, we aim to elaborate a model of BEC loaded in a 2D planar harmonic-potential (HO) trap applied in the plane of $(x, y)$, combined with competing HO trap $\sim z^{2}$ and singular repulsive potential $\sim$ $1 / z^{2}$ acting along the perpendicular axis. This scheme shapes the condensate into a double-pancake configuration parallel to the plane of $z=0$, as shown in Fig 1. Then, using the technique similar to that elaborated in Ref. [57], we derive an effective 2D equation governing the system's planar dynamics. Note that, differently from the attractive potentials $\sim-1 / r^{2}$, considered in works [33, 80-84] (see also a brief review in [85]), which 

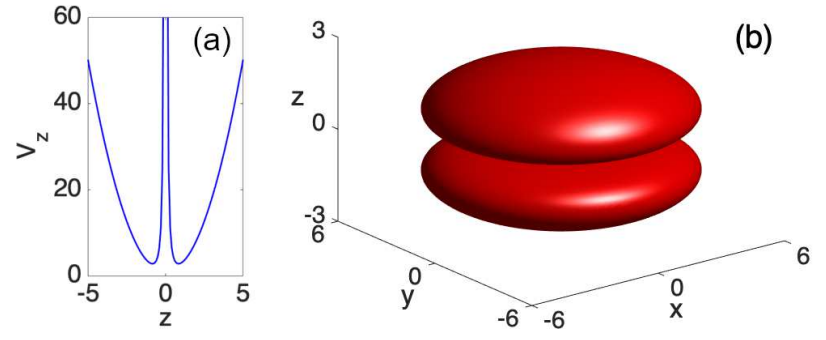

FIG. 1. (a) The confining transverse potential, $V_{z}=\zeta^{2} / z^{2}+$ $2 z^{2}$, from Eq. (3). (b) The 3D isosurface of the local density, $|\psi(x, y, z)|^{2}=0.003$, corresponding to the GS (ground-state) solution of Eq. (1) with the repulsive sign $(g=-0.1)$ of the nonlinearity and potential given by Eqs. (3) and (4), with $\lambda=$ 0.1 and $\zeta=1$.

may absorb atoms through the mechanism of the quantum collapse (alias "fall onto the center" [86]), the total number of atoms is maintained constant, in the case of the repulsive singular potential.

The rest of the paper is organized as follows. In the next section we present the model, including a possibility of its physical realization, and derive an effective NPSE for it. This section also contains exact analytical results for bound states and a spectrum produced by the singular trapping potential, and for a quantum phase transition between the $\mathrm{HO}$ potential and the one which includes the singular term. In Sec. III we check the accuracy of the effective 2D model, by comparing its predictions with results produced by the full threedimensional GPE. This is done for static and dynamical states alike, including the ground state (GS) and vortex modes. Stability and instability of vortices with topological charges $S=1,2$, and 3 is also addressed in Sec. III. The paper is concluded by Sec. IV.

\section{THE EFFECTIVE TWO-DIMENSIONAL NPSE (NONPOLYNOMIAL SCHRÖDINGER EQUATION) AND ANALYTICAL CONSIDERATIONS}

\section{A. Basic equations}

We start by considering the 3D GPE for atomic BEC, written in the usual scaled form $[87,88]$ :

$$
i \frac{\partial \psi}{\partial t}=-\frac{1}{2} \nabla^{2} \psi+V \psi+2 \pi g|\psi|^{2} \psi,
$$

where $\psi=\psi(x, y, z, t)$ is the mean-field wave function with the integral norm set equal to 1 ,

$$
\iiint|\psi(x, y, z)|^{2} d x d y d z=1,
$$

$V(x, y, z)$ is a trapping potential, and $g=2 N a_{s} / a_{z}$ is the strength of the two-body interatomic interaction, with $N$ being the number of atoms, while $a_{S}$ and $a_{z}$ are, respectively, the s-wave scattering length of atomic collisions and the confinement length of the $\mathrm{HO}$ potential acting in the direction perpendicular to the system's plane, which is adopted as the unit of length. Attractive and repulsive interactions correspond, respectively, to $g<0$ and $g>0$.

In this work, we assume a combination of strong transverse and relatively weak planar potentials, viz.,

$$
V(x, y, z)=\left(\frac{\zeta^{2}}{z^{2}}+2 z^{2}\right)+U(x, y) .
$$

The latter term is chosen as the isotropic HO,

$$
U(x, y)=\frac{1}{2} \lambda^{2}\left(x^{2}+y^{2}\right),
$$

with strength $\lambda^{2}$, while term $2 z^{2}$ in the transverse potential represents the usual one-dimensional HO trap $[87,88]$, with the strength normalized with the help of the scaling invariance of Eq. (1).

The shape of the transverse potential, as defined by Eq. (3), is displayed in Fig. 1 (a). As concerns the singular repulsive term in the transverse part of the potential in Eq. (3), with scaled strength $\zeta^{2}$, it may represent a specifically designed physical setting. Indeed, the repulsive action on cold atoms may be exerted by a nearlyresonant blue-detuned optical field with frequency $\omega$, close to frequency $\omega_{0}$ of atomic dipole oscillations (see, e.g., Refs. $[89,90]$ and references therein), the respective interaction energy being proportional to $\left(\omega^{2}-\omega_{0}^{2}\right)^{-1}$. This dependence may be used to induce an effective repulsion potential, imposing spatial modulation on $\omega_{0}^{2}$ by means of the Zeeman effect [86] in a spatially inhomogeneous dc magnetic field, or quadratic Stark - Lo Surdo effect [86] in an electrostatic field, cf. Ref. [91], where an inhomogeneous field was used to design spatially modulated dipole-dipole repulsion in BEC. In the present context, the field should be shaped so as to make $\omega_{0}^{2}(z)=\omega^{2}-\Omega^{2} z^{2}$, which leads to the singular term in Eq. (4) with $\zeta^{2} \sim 1 / \Omega^{2}$. In particular, in the case of the Zeeman effect, the necessary spatial maximum or minimum of the magnetic field at $z=0$ may be created by means of a solenoid shaped, respectively, as a hyperboloid or "barrel". In physical units, the value of $\zeta$ relevant for the experimental realization is estimated as $\sim 50^{-} \mathrm{m}^{2}$. If the realization is based on the Zeeman effect, the creation of nonuniform magnetic field with the respective values of the variation length is possible (see, e.g., Ref. [92]).

The use of the transverse potential given by Eq. (3) is relevant for several reasons. First, as shown in detail, below, it offers a relatively simple setting for the realization of a quantum phase transition, and also gives rise to specific spectrum of excitations, which may be effects interesting for the experimental realization. In addition, the potential splits the 3D space in two uncommunicating subspaces, each one admitting its own states, which thus suggests a possibility to create "quantum chimeras" under the action of this potential. 

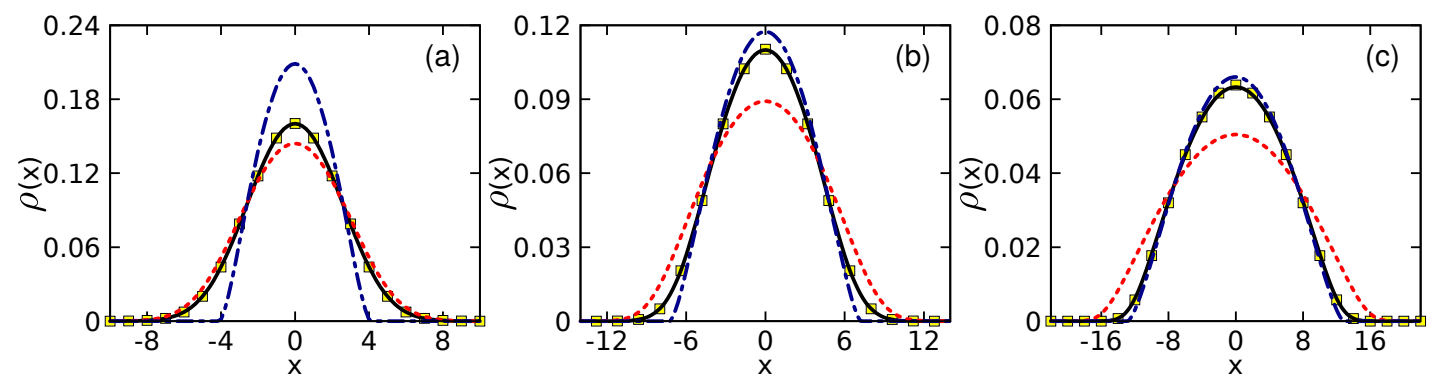

FIG. 2. Normalized density profile $\rho(x)$ in the central cross section (drawn through $y=0$ ) of the 2D GS for the repulsive BEC with nonlinearity strength $g=1(\mathrm{a}), g=10(\mathrm{~b})$, and $g=100(\mathrm{c})$, in the presence of the in-plane potential (4) with $\lambda=0.1$ and $\zeta=1$. The corresponding density profiles for the full 3D GPE (1), 2D NPSE (29), 2D cubic NLSE (32), and TFA (35) are plotted by chains of yellow squares, black solid lines, red dashed lines, and blue dashed-dotted lines, respectively.

B. The transverse wave function: analytical results and the quantum phase transition

The separation of the strong transverse and weak planar potentials in Eq. (3) suggests that the reduction 3D $\rightarrow 2 \mathrm{D}$ may be facilitated by the adoption of the factorized ansatz for the 3D wave function,

$$
\psi(x, y, z, t)=\chi(z, t) \Phi(x, y, t) .
$$

First, the substitution of this ansatz in Eq. (1), keeping only the strong transverse potential and time derivative, leads to the 1D linear Schrödinger equation,

$$
i \frac{\partial \chi}{\partial t}=\hat{H}_{z} \chi \equiv-\frac{1}{2} \frac{\partial^{2} \chi}{\partial z^{2}}+\left(\frac{\zeta^{2}}{z^{2}}+2 z^{2}\right) \chi .
$$

It is easy to find an exact solution for the GS of this equation,

$$
\begin{gathered}
\chi_{0}(z, t)=\frac{2^{(1 / 2)(\alpha+1 / 2)}}{\sqrt{\Gamma(\alpha+1 / 2)}}|z|^{\alpha} \exp \left(-i \mu_{z}^{(0)} t-z^{2}\right), \\
\alpha=1 / 2+\sqrt{1 / 4+2 \zeta^{2}}, \\
\mu_{z}^{(0)}=2+\sqrt{1+8 \zeta^{2}},
\end{gathered}
$$

where $\Gamma$ is the Gamma-function, and the constant coefficient is determined by the normalization condition,

$$
\int_{-\infty}^{+\infty}|\chi(z)|^{2} d z=1
$$

Note that, in particular, $\alpha=2$ for $\zeta^{2}=1$, and $\alpha=3$ for $\zeta^{2}=3$. It is worthy to note that the singular term, $\sim \zeta^{2} / z^{2}$, in the integral which produces the GS energy,

$$
\int_{-\infty}^{+\infty} \chi \hat{H}_{z} \chi d x=\mu_{z}
$$

with Hamiltonian $\hat{H}_{z}$ defined by Eq. (6), converges if the wave function (7) is substituted in Eq. (11).
There is another formal solution to Eq. (6), represented by Eq. (7) with $\alpha$ replaced by

$$
\tilde{\alpha}=1 / 2-\sqrt{1 / 4+2 \zeta^{2}}
$$

and a nominally lower eigenvalue,

$$
\tilde{\mu}_{z}=2-\sqrt{1+8 \zeta^{2}},
$$

instead of the values given by Eqs. (8) and (9). However, this solution is physically irrelevant, as the wave function is singular at $z=0$ [and non-normalizable at $\zeta^{2} \geq 3 / 8$, which corresponds to divergent integral in Eq. (10) and $\left.\tilde{\mu}_{z}<0\right]$, except for the limit case of $\zeta=0$, when the solution based on Eqs. (12) and (13) corresponds to the commonly known GS of the HO potential, while the above solution, with $|z|$ replaced by $z$ in Eq. (7), produces the first excited state. Furthermore, the actual energy of the singular state, as given by the integral expression (11), diverges for all $\zeta^{2}>0$, breaking the equality of the integral expression to $\mu_{z}$, Thus, there is a strong discontinuity (quantum phase transition) in the spectrum of bound states produced by Hamiltonian $\mathrm{H}_{z}$ in Eq. (6), as there is a jump of the GS, following the introduction of an arbitrarily small value of $\zeta^{2}$.

The transverse potential displayed in Fig. 1(a) seems as a structure built of two symmetric potential wells separated by a tall barrier. This configuration is used for the consideration of spontaneous symmetry breaking of optical and matter waves in various nonlinear photonic and BEC settings [93]. However, the singular potential barrier $\sim z^{-2}$ is so strong that it splits the system in two non-communicating half-spaces (an effect known as "superselection" [94]), therefore the consideration of Eq. (1) with potential (3) on the entire axis, $-\infty<z<+\infty$, amounts to solving the same problem on the half-axis, $0 \leq z<\infty$. Indeed, solutions (7) with all values $\zeta^{2}>0$ satisfy boundary conditions $\chi=d \chi / d z=0$ at $z=0$, therefore any two different solutions, built independently at $z>0$ and $z<0$, may be matched at point $z=0$. This fact implies that, in the one hand, the symmetry-breaking phenomenology becomes trivial in 
the present setting, but, on the other hand, it opens a possibility to construct complexes of completely different states, which would seem as "quantum chimeras", cf. Refs. $[95,96]$.

In the same vein, it is interesting to compare the singular potential (7) to one featuring a more general singularity, viz.,

$$
V_{z}=\frac{\zeta^{2}}{|z|^{h}}+2 z^{2}
$$

with $h>0$. It is easy to see that, at $h<2$, the expansion of the wave function at $|z| \rightarrow 0$ is

$$
\chi \approx \text { const } \cdot e^{-i \mu t}\left[1+\frac{2 \zeta^{2}}{(2-h)(1-h)}|z|^{2-h}\right],
$$

except for the case of $h=1$, when Eq. (15) is replaced by

$$
\chi \approx \text { const } \cdot e^{-i \mu t}\left[1+2 \zeta^{2}|z| \ln (|z|)\right],
$$

The regular dependence of the expansions in Eqs. (15) and (16) on $\zeta^{2}$ means that the change $\zeta^{2}=0 \rightarrow \zeta^{2}>0$ does not lead to a phase transition. On the other hand, in the case of the singular potential (14) with $h>2$ the asymptotic form of the wave function at $z \rightarrow 0$ is drastically different:

$$
\chi \approx \text { const } \cdot e^{-i \mu t} \exp \left(-\frac{2 \sqrt{2 \zeta^{2}}}{(h-2)|z|^{(h-2) / 2}}\right),
$$

which definitely implies that a strong phase transition takes place. Thus, the case of $h=2$ in Eq. (14), addressed in the present work, is a critical one, in which the quantum phase transition commences.

Getting back to Eq. (6), it is relevant to mention that the first excited state of this Hamiltonian can also be found in an exact form:

$\chi_{1}=$ const $\cdot|z|^{\alpha}\left(1-\frac{4 z^{2}}{2+\sqrt{1+8 \zeta^{2}}}\right) \exp \left(-i \mu_{z}^{(1)} t-z^{2}\right)$,

$$
\mu_{z}^{(1)}=6+\sqrt{1+8 \zeta^{2}},
$$

where $\alpha$ is the same as given by Eq. (8). Moreover, it is easy to find an exact full spectrum of eigenvalues for higher-order excited states, with number $n$ (in the limit of $\zeta^{2} \rightarrow 0$, the spectrum carries over into energy eigenvalues of $\mathrm{HO}$ with odd numbers, $n_{\mathrm{OH}} \equiv 1+2 n$ ):

$$
\mu_{z}^{(n)}=2(1+2 n)+\sqrt{1+8 \zeta^{2}}, n=0,1,2, \ldots .
$$

Note that this series of eigenvalues is equidistant, similar to the $\mathrm{OH}$ spectrum.

The formal eigenvalue given by Eq. (13) also generates an infinite series of higher-order ones, $\tilde{\mu}_{z}^{(n)}=$
$2(1+2 n)-\sqrt{1+8 \zeta^{2}}$, which are formal counterparts of $\mathrm{OH}$ eigenvalues corresponding to even states, but all the respective wave functions are singular, i.e., unphysical. Eigenvalues $\tilde{\mu}_{z}^{(n)}$ are counterparts of those of HO with even numbers, $n_{\mathrm{OH}} \equiv 2 n$.

Lastly, it is also worthy to note that the $2 \mathrm{D}$ version of Eq. (6), i.e.,

$$
\begin{aligned}
i \frac{\partial \chi_{2 \mathrm{D}}}{\partial t} & =-\frac{1}{2}\left(\frac{\partial^{2}}{\partial r^{2}}+\frac{1}{r} \frac{\partial}{\partial r}+\frac{1}{r^{2}} \frac{\partial^{2}}{\partial \theta^{2}}\right) \chi_{2 \mathrm{D}} \\
& +\left(\frac{\zeta^{2}}{z^{2}}+2 z^{2}\right) \chi_{2 \mathrm{D}},
\end{aligned}
$$

where $(r, \theta)$ are polar coordinates in the 2D plane, produces exact solutions with the azimuthal quantum number, alias vorticity, $l=0,1,2, \ldots(l=0$ corresponds to the GS):

$$
\begin{aligned}
& \chi_{2 \mathrm{D}}^{(l)}=\mathrm{const} \cdot r^{\eta} \exp \left(-i \mu_{2 \mathrm{D}}^{(l)} t+i l \theta-r^{2}\right), \\
& \eta=\sqrt{2 \zeta^{2}+l^{2}}, \mu_{2 \mathrm{D}}^{(l)}=2\left(\sqrt{2 \zeta^{2}+l^{2}}+1\right) .
\end{aligned}
$$

Note that the spectrum of the 2D eigenvalues, given by Eq. (23), is not equidistant, unlike its 1D counterpart (20).

\section{Derivation of the two-dimensional equation}

The spatial-dimension reduction 3D $\rightarrow$ 2D proceeds via the variational approach, which is based on the Lagrangian density corresponding to Eq. (1) with potential (3):

$$
\begin{gathered}
\mathcal{L}=\frac{i}{2}\left(\psi^{*} \frac{\partial \psi}{\partial t}-\psi \frac{\partial \psi^{*}}{\partial t}\right)-\frac{1}{2}|\nabla \psi|^{2}-\left(\frac{\zeta^{2}}{z^{2}}+2 z^{2}\right)|\psi|^{2} \\
-U(x, y)|\psi|^{2}-\pi g|\psi|^{4}
\end{gathered}
$$

We use the factorized ansatz (5), in which all the time dependence is included in the planar wave function, $\Phi$, and the transverse one is adopted in the form suggested by solution (7), except for the phase factor, $\exp \left(-i \mu_{z}^{(0)} t\right)$ :

$$
\psi=[\Gamma(\alpha+1 / 2)]^{-1 / 2}\left(\frac{\sqrt{2}}{\sigma}\right)^{\alpha+1 / 2}|z|^{\alpha} \exp \left(-\frac{z^{2}}{\sigma^{2}}\right) \Phi,
$$

where $\alpha$ is defined by Eq. (8), and $\sigma=\sigma(x, y, t)$ is introduced as a variational parameter accounting for possible evolution of the transverse-confinement size. Stationary states generated by ansatz (25) imply the doublepancake shape in the 3D space, with $\psi(z=0)=0$, and a pair of symmetric maxima of density $|\psi(x, y, z)|^{2}$ at

$$
z_{\max }^{2}=(\alpha / 2) \sigma^{2}
$$


see Fig. 1 (b) as an illustration. Note also that ansatz (25) is defined so that the transverse factor is subject to the unitary normalization [cf. Eq. (10)], hence it follows from Eq. (2) that the 2D wave function also has its norm equal to 1 :

$$
\iint|\Phi(x, y, t)|^{2} d x d y=1
$$

Next, by inserting ansatz (25) in the Lagrangian density (24), performing the integration in the transverse direction, and neglecting the spatial derivatives of $\sigma$ (in the adiabatic approximation, cf. Ref. [57]), one can derive the corresponding effective Lagrangian:

$$
\begin{aligned}
& \mathcal{L}_{\text {eff }}=\frac{i}{2}\left(\Phi^{*} \frac{\partial \Phi}{\partial t}-\Phi \frac{\partial \Phi^{*}}{\partial t}\right)-\frac{1}{2}\left|\nabla_{\perp} \Phi\right|^{2}-\left(a+\frac{1}{2}\right) \times \\
& \left(\sigma^{2}+\frac{1}{\sigma^{2}}\right)|\Phi|^{2}-U(x, y)|\Phi|^{2}-\frac{\pi}{2^{2 \alpha}} \frac{\Gamma(2 \alpha+1 / 2)}{\Gamma^{2}(\alpha+1 / 2)} g \frac{|\Phi|^{4}}{\sigma},
\end{aligned}
$$

where $\nabla_{\perp}$ is the gradient operator in Cartesian coordinates $(x, y)$. This expression gives rise to the EulerLagrange equations:

$$
\begin{gathered}
i \frac{\partial \Phi}{\partial t}=-\frac{1}{2} \nabla_{\perp}^{2} \Phi+U(x, y) \Phi+\left(\alpha+\frac{1}{2}\right) \times \\
\left(\sigma^{2}+\frac{1}{\sigma^{2}}\right) \Phi+\frac{\pi}{2^{2 \alpha-1}} \frac{\Gamma(2 \alpha+1 / 2)}{\Gamma^{2}(\alpha+1 / 2)} g \frac{|\Phi|^{2}}{\sigma} \Phi \\
\sigma^{4}-\frac{\pi}{2^{2 \alpha}(2 \alpha+1)} \frac{\Gamma(2 \alpha+1 / 2)}{\Gamma^{2}(\alpha+1 / 2)} g|\Phi|^{2} \sigma-1=0
\end{gathered}
$$

The 2D nonpolynomial Schrödinger equation (NPSE), Eq. (29), is the main result of the derivation. It determines the density profile in the 2D plane, taking into regard effects of the transverse BEC structure.

First, it is natural to consider the low-density limit of Eq. (29), i.e., with $g|\Phi|^{2} \ll 1$ and $\sigma$ close to 1 , as it follows from Eq. (30):

$$
\sigma-1 \approx \frac{\pi \Gamma(2 \alpha+1 / 2)}{2^{2 \alpha+1}(2 \alpha+1) \Gamma^{2}(\alpha+1 / 2)} g|\Phi|^{2} .
$$

The small difference of $\sigma$ from 1 , given by Eq. (31), produces no contribution to NPSE (29) in the lowest approximation, hence this equation amounts to the usual nonlinear Schrödinger equation (NLSE) with the cubic term:

$$
\begin{aligned}
i \frac{\partial \Phi}{\partial t} & =-\frac{1}{2} \nabla_{\perp}^{2} \Phi+U(x, y) \Phi+(2 \alpha+1) \Phi \\
& +\frac{\pi}{2^{2 \alpha-1}} \frac{\Gamma(2 \alpha+1 / 2)}{\Gamma^{2}(\alpha+1 / 2)} g|\Phi|^{2} \Phi .
\end{aligned}
$$

In the opposite high-density limit with the repulsive sign of the nonlinearity, $g>0$, it is natural to apply the Thomas-Fermi approximation (TFA) to the underlying three-dimensional GPE (1), neglecting the kineticenergy term (second derivatives) in it. In the absence of kinetic energy, one has additional scale invariance, which makes it possible to fix $\zeta^{2}=1$ in Eq. (3), keeping the unitary normalization of the wave function, by defining

$$
z=\sqrt{\zeta} z^{\prime}, t=t^{\prime} / \zeta, \psi=\zeta^{-1 / 4} \psi^{\prime}, \lambda=\sqrt{\zeta} \lambda^{\prime}, g=\zeta^{3 / 2} g^{\prime} .
$$

Then, dropping the primes, the TFA may be naturally based on the following ansatz:

$$
\psi(x, y, z, t)=\frac{4}{\sqrt{3}}\left(\frac{2}{\pi}\right)^{1 / 4} z^{2} \exp \left(-z^{2}-i \mu t\right) \Phi_{\mathrm{TF}}
$$

cf. Eq. (25), where an expression for the 2D wave function is derived by substituting this ansatz in the 3D equation (1), dropping the second derivatives in it, and integrating the resultant equation along coordinate $z$ :

$$
\left|\Phi_{\mathrm{TF}}\right|^{2}= \begin{cases}\frac{27 \sqrt{6}}{80 \sqrt{\pi} g}[\mu-5-U(x, y)], & \text { at } \mu>U(x, y)+5 \\ 0, & \text { at } \mu \leq U(x, y)+5\end{cases}
$$

Finally, the respective chemical potential, $\mu_{\mathrm{TF}}$, can be obtained from the normalization condition,

$$
\iint\left|\Phi_{\mathrm{TF}}(x, y)\right|^{2} d x d y=1
$$

as per Eq. (27).

\section{NUMERICAL RESULTS}

GS solutions of the 2D and 3D equations addressed in this work were produced by means of the well-known method of the imaginary-time evolution [97]. It was realized by means of a split-step scheme, based on the Crank-Nicolson algorithm, with space and time steps $\Delta_{x}=0.04$ and $\Delta_{t}=0.001$, respectively, in 2D and 3D cases alike. For details of conducting numerical simulations of this type see, e.g., Refs. $[98,99]$. To check the accuracy of the effective 2D NPSE (29), results produced by this equation, as well as those provided by the $2 \mathrm{D} \mathrm{cu}-$ bic NLSE (32), and the TFA based on Eq. (35), were compared to those obtained from the numerical solution of the full 3D GPE (1). Below, we report the results for the GS and vortex modes, in both cases of the repulsive and attractive nonlinearity, i.e., $g>0$ and $g<0$. 


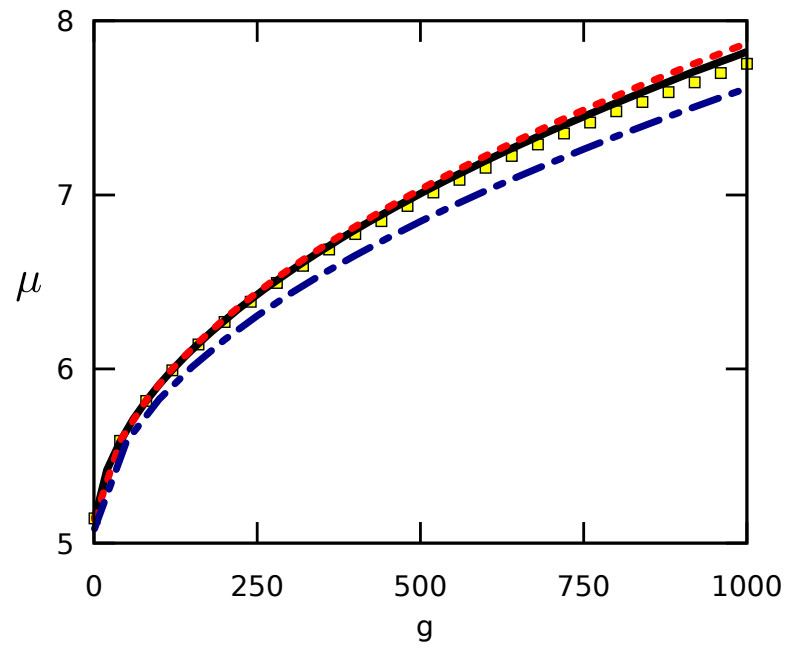

FIG. 3. Chemical potential $\mu$ of the GS (ground state), trapped in the in-plane potential (4) with $\lambda=0.1$ and $\zeta=1$, versus the self-repulsion strength, $g$, Displayed are numerical results obtained from the full 3D GPE, effective 2D NPSE, 2D cubic NLSE, and TFA, under normalization conditions (2), (27) and (36), respectively. Symbols and curves have the same meaning as in Fig. 2.
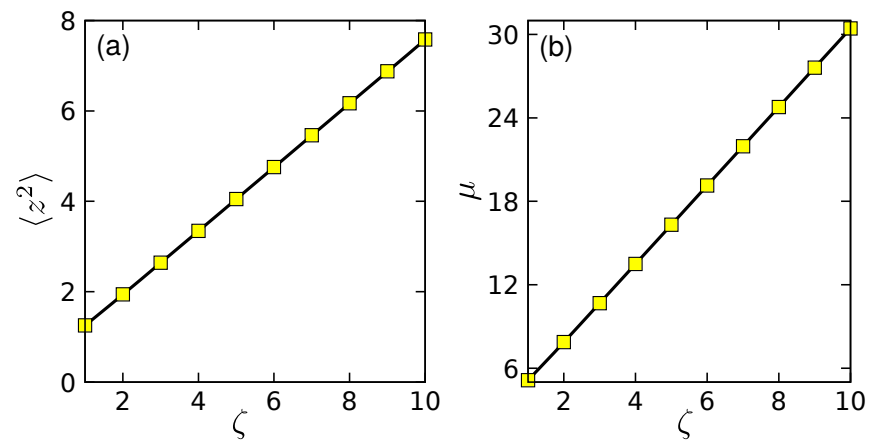

FIG. 4. (a) Mean-squared axial length $\left\langle z^{2}\right\rangle$ and (b) chemical potential $\mu$ of the GS versus the scaled strength $\zeta$ of the transverse potential barrier. The GS is trapped in the in-plane potential (4), with $\lambda=0.1$. The numerical results produced by the full 3D-GPE are displayed by yellow squares, and those obtained from the effective 2D NPSE are represented by black solid lines. The results were generated with normalization conditions (2) and (27), respectively.

\section{A. GS (ground-state) solutions}

\section{The repulsive nonlinearity}

Here, we compare the 1D lumped density profile produced by the full 3D equation (1) for the stationary GS,

$$
\rho(x)=\iint|\psi(x, y, z, t)|^{2} d y d z
$$

with its counterpart obtained from the $2 \mathrm{D}$ equations (29), (32), and (35), calculated as

$$
\rho(x)=\int_{-\infty}^{+\infty}|\Phi(x, y, t)|^{2} d y
$$

We start the numerical analysis by considering the repulsive nonlinearity $(g>0)$ in the presence of the 2D (in-plane) $\mathrm{HO}$ trapping potential. Note that the configuration of the BEC can be defined as double-pancakeshaped if the transverse confinement is much tighter than the in-plane potential, i.e., $\lambda^{2} \ll \alpha^{-4}$, see Eq. (26).

In Fig. 2 we display in-plane density profiles of the GS in the repulsive condensate $(g>0)$ under the action of potential (4), for three different values of $g$. In comparison to the full 3D GPE, the 2D NPSE provides virtually exact results, while the low-density limit and TFA, based on Eqs. (32) and (35), respectively, produce visible discrepancies. In particular, for the case of relatively weak nonlinearity, with $g=1$, displayed in Fig. 2 $(\mathrm{a})$, at the central point $(x=0)$ the error between the numerically exact value of the density, obtained from the full 3D GPE equation, and the 2D approximations is $\simeq 0.05 \%, \simeq 10 \%$, and $\simeq 30 \%$ for the $2 \mathrm{D}$ NPSE, cubic 2D NLSE, and TFA, respectively (naturally, TFA is irrelevant in the case of weak nonlinearity). In the opposite case of strong nonlinearity $(g=100)$, shown in Fig. 2 (c), the same percentage errors are $\simeq 0.9 \%, \simeq 21 \%$, and $\simeq 3 \%$ (in this case, the TFA is quite relevant, while the low-density approximation is not). Note that the error produced by the 2D NPSE increases with the increase of $g$, remaining, nevertheless, fairly small. Similar to the situation considered in Ref. [57], this happens because ansatz (25), used for the $3 \mathrm{D} \rightarrow 2 \mathrm{D}$ reduction method, is taken as a solution of Eq. (7) with $g=0$, thus getting less accurate with the increase of $g$.

Another way of evaluating the accuracy of the 2D NPSE is through the calculation of chemical potential $\mu$, setting $\psi(x, y, t)=\psi(x, y) \exp (-i \mu t)$ and $\Phi(x, y, t)=$ $\Phi(x, y) \exp (-i \mu t)$ in Eqs. (29) and (32), while, as mentioned above, $\mu_{\mathrm{TF}}$ is defined by normalization condition (36). Figure 3 shows that the chemical potentials obtained from the 3D GPE and 2D NPSE always stay very close, while the low-density approximation and TFA produce discrepancies. Note that the positive slope of the $\mu(g)$ dependence is tantamount to $d \mu / d N>0$ , if $g$ is kept constant, while norm $N$ is not fixed by Eq. (27) but is allowed to vary. In turn, the latter condition (the anti-Vakhitov-Kolokolov criterion) is necessary for stability of localized states under the action of selfrepulsion [100]. In fact, for simple modes, such as GS, this criterion may be sufficient for the stability, which is confirmed by direct simulations of their perturbed evolution (not shown here in detail).

We also analyzed the efficiency of the 2D NPSE with respect to variation of the transverse scaled strength $\zeta$. To this end, we set $\lambda=0.1$ and $g=1$, and calculate the 

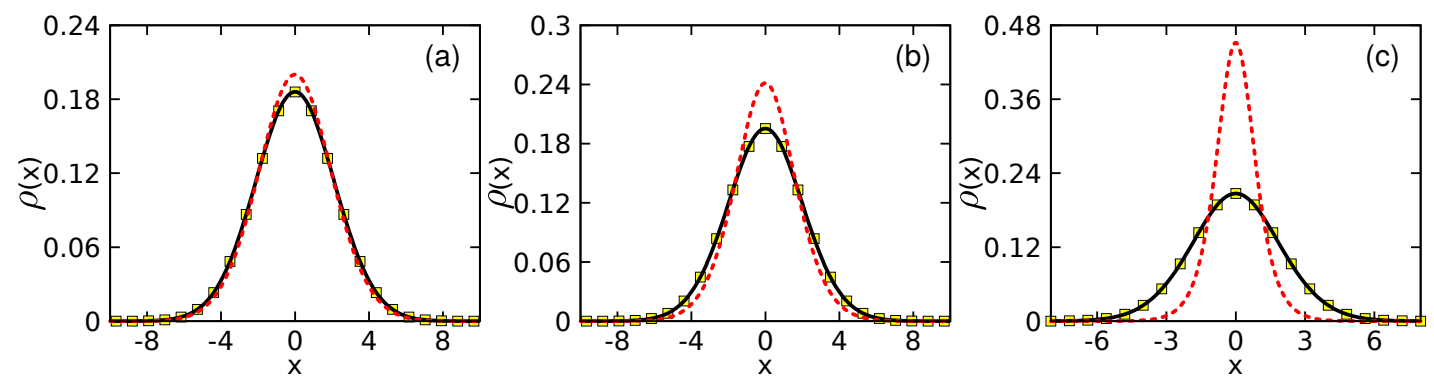

FIG. 5. The cross section of 2D density profiles $\rho(x)$ for the GS in the attractive condensate with $g=-0.3(\mathrm{a}), g=-0.6$ (b), and $g=-0.9$ (c), in the presence of the in-plane trapping potential (4) with $\lambda=0.1$ and $\zeta=1$. The results produced by the full 3D GPE, 2D NPSE, and 2D cubic NLSE are shown, severally, by yellow squares, black solid lines, and red dashed lines.

mean squared axial length,

$$
\left\langle z^{2}\right\rangle=\iiint \frac{2^{\alpha+1 / 2}|z|^{2(\alpha+1)} e^{-2 z^{2} / \sigma^{2}}}{\Gamma(\alpha+1 / 2) \sigma^{2 \alpha+1}}|\Phi(x, y, t)|^{2} d x d y d z
$$

based on the ansatz (25) for the 2D NPSE, which we compare to $\left\langle z^{2}\right\rangle=\iiint z^{2}|\psi(x, y, z)|^{2} d x d y d z$, calculated as per the 3D GPE. This result is shown in Fig. 4(a), where we see that the effective equation maintains its accuracy with the increase of $\zeta$. Corroborating this result, in Fig. 4 (b) we present the behavior of the chemical potential $\mu(\zeta)$, in which the linear dependence $\mu(\zeta)$, produced by the 2D NPSE, is virtually overlapped with that produced by the 3D GPE.

\section{The attractive nonlinearity}

Next, we address the case of the self-attraction, $g<$ 0 . For this case, in Fig. 5 we plot cross-sections of the 2D density profiles, as produced by the full 3D GPE, 2D NPSE, and cubic 2D NLSE (the TFA is irrelevant for the attractive nonlinearity). Similar to the case of $g>0$, the 2D NPSE predicts the profiles in a virtually exact form, while the cubic 2D NLSE produces a visible discrepancy with the increase of $|g|$.

Furthermore, Eq. (29) with $g<0$ gives rise to the collapse of the wave function, when the strength of the self-attraction exceeds a critical value, $|g|>\left|g_{c}\right|$, as may be expected in the 2D setting [101]. We have found the value of $g_{c}$ for the attractive BECs in the presence of the in-plane trapping potential (4), under normalization conditions (2) and (27), respectively, with $\lambda=0.1$, using the full 3D GPE, as well as the other approximations. We have thus obtained $g_{c}=-2.1$ from the 2D NPSE, which is exactly the same as produced by the full 3D GPE. On the other hand, in the framework of the cubic 2D NLSE the collapse occurs at $g_{c}=-2.5$.

\section{B. Vortex modes under the action of the repulsive and attractive nonlinearity}

Here we address vortex solutions produced by the full $3 \mathrm{D}$ and effective $2 \mathrm{D}$ equations. To this end, the $3 \mathrm{D}$ wave function is looked for, in the cylindrical coordinates, as $\psi(r, \theta, z, t)=\Psi(r, z, t) \exp (i S \theta)$, resulting in the following equation:

$$
\begin{aligned}
i \frac{\partial \Psi}{\partial t} & =-\frac{1}{2}\left[\frac{\partial^{2} \Psi}{\partial r^{2}}+\frac{\partial^{2} \Psi}{\partial z^{2}}+\frac{1}{r} \frac{\partial \Psi}{\partial r}\right]+\frac{S^{2}}{r^{2}} \Psi \\
& +\left(\frac{\zeta^{2}+2 z^{4}}{z^{2}}+\frac{1}{2} \lambda^{2} r^{2}\right) \Psi+\left.2 \pi g\right|^{2} \Psi
\end{aligned}
$$

where $S$ is integer vorticity. Similarly, substituting $\Phi(r, \theta, t)=\phi(r, t) \exp (i S \theta)$ in the 2D NPSE equation (29) leads to the radial equation

$$
\begin{aligned}
i \frac{\partial \phi}{\partial t} & =-\frac{1}{2}\left[\frac{\partial^{2} \phi}{\partial r^{2}}+\frac{1}{r} \frac{\partial \phi}{\partial r}\right]+\left(\alpha+\frac{1}{2}\right)\left(\sigma^{2}+\frac{1}{\sigma^{2}}\right) \phi \\
& +\frac{1}{2} \lambda^{2} r^{2} \phi+\frac{S^{2}}{r^{2}} \phi+\frac{\pi}{2^{2 \alpha-1}} \frac{\Gamma(2 \alpha+1 / 2)}{\Gamma^{2}(\alpha+1 / 2)} g \frac{|\phi|^{2}}{\sigma} \phi,
\end{aligned}
$$

which is combined with Eq. (30).

In Fig. 6, we display examples of radial density profiles of the vortex modes produced by Eqs. (40) and (41), with $\rho(r)=\int_{-\infty}^{+\infty}|\Psi(r, z, t)|^{2} d z$ and $\rho(r)=|\phi(r, t)|^{2}$, respectively. The profiles are presented for both $g<0$ and $g>0$, and for three values of the vorticity, $S=1,2,3$. The results clearly corroborate the accuracy of the $2 \mathrm{D}$ NPSE in describing the vortex states of the 3D GPE.

The dimensional reduction method employed here, which is based on ansatz (25), can also be used to produce lumped density profiles of the condensate in the axial direction, by the integration in the $(x, y)$ plane:

$$
\rho(z)=\frac{2^{\alpha+3 / 2} \pi}{\Gamma(\alpha+1 / 2)}|z|^{2 \alpha} \int_{0}^{\infty} \exp \left(-2 \frac{z^{2}}{\sigma^{2}}\right) \frac{|\phi(r, t)|^{2}}{\sigma^{2 \alpha+1}} r d r .
$$

It is relevant to compare this approximate result to its counterpart, i.e., the integrated density, provided by the 

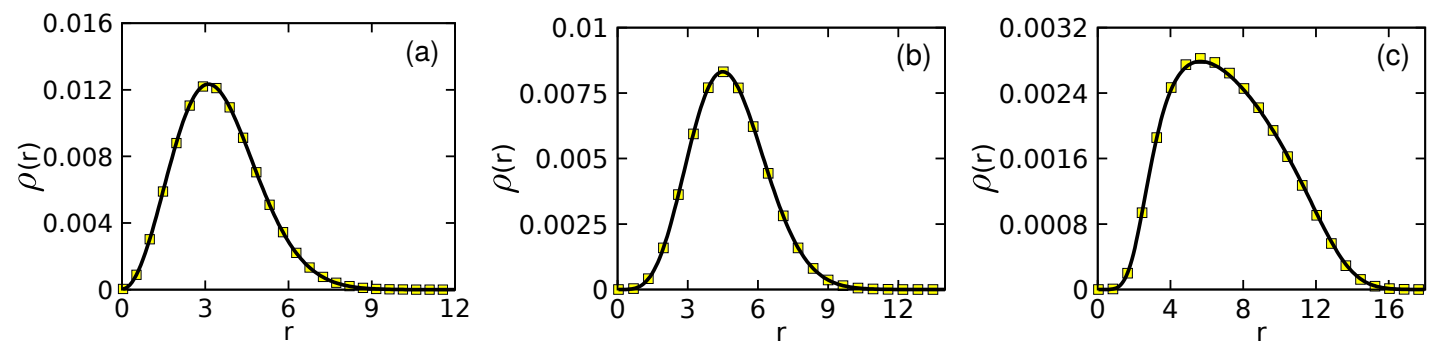

FIG. 6. Radial density profiles $\rho(r)$ for states with vorticity $S$ trapped in the in-plane potential (4) with $\lambda=0.1$ and $\zeta=1$, pertaining to: (a) $S=1$ and $g=-0.8$, (b) $S=2$ and $g=1$, and (c) $S=3$ and $g=100$. The profiles obtained from the full 3D GPE and 2D NPSE are displayed, severally, by yellow squares and black solid lines.

full 3D GPE as

$$
\rho(z)=2 \pi \int_{0}^{\infty}|\Psi(r, z, t)|^{2} r d r
$$

The comparison, presented in Fig. 7(a) for typical axial profiles with $S=2$, again demonstrates that the $2 \mathrm{D}$ NPSE offers virtually exact results, i.e., it can be reliably used for the full description of the zero-vorticity and vortex modes. In particular, it demonstrates that the radius of the central "hole" in the trapped mode, induced by the embedded vorticity, increases with the growth of $S$, as can be clearly seen in Fig. 7(b). This is a general property of solitons with embedded vorticity, which admits an analytical explanation [102]. On the other hand, the axial density profile is very weakly affected by the value of $S$, as shown by Fig. 7(c).

Addressing the onset of the collapse in vortex states with $S=1,2$ and 3, confined by the in-plane trapping potential (4) with $\lambda=0.1$, we have computed a set of respective critical values of the self-attraction coupling constant, $g_{c}$, for the vortices subject to normalization conditions (2) and (27). These results were produced by the full 3D GPE, as well as by means of the 2D NPSE and cubic NLSE, which are collected in Table I, where we have also included the result for the GS $(S=0)$, presented in the previous subsection. One can see that the 2D NPSE produces accurate predictions, in comparison to those found from the full 3D GPE for all values of $S$. Note that $g_{c}$ strongly increases with the growth of $S$, similar to what was observed in other models [103].

\section{Stability of the vortex modes with $S=1,2,3$}

To conclude the analysis of the vortex solutions under the action of the cubic self-attraction, we studied their stability by means of direct simulations, starting from inputs perturbed by anisotropic deformations, which may readily initiate splitting of unstable vortices in nonlinear models [103-106]. For this purpose, we used, first, 2D NPSE (29) with the in-plane trapping potential (4). The anisotropically deformed initial condition with vor-

\begin{tabular}{|c|c|c|c|}
\hline$S$ & $\left(g_{c}\right)_{3 \mathrm{D}-\mathrm{GPE}}$ & $\left(g_{c}\right)_{2 \mathrm{D}-\mathrm{NPSE}}$ & $\left(g_{c}\right)_{\text {cubic } 2 \mathrm{D}-\mathrm{NLSE}}$ \\
\hline \hline 0 & -2.1 & -2.1 & -2.5 \\
\hline 1 & -8.0 & -8.1 & -9.7 \\
\hline 2 & -12.7 & -13.2 & -18.4 \\
\hline 3 & -16.1 & -17.0 & -27.1 \\
\hline
\end{tabular}

TABLE I. Critical values of the strength of the two-body interatomic interaction $g_{c}$ for the onset of the collapse, calculated for the ground $(S=0)$ and vortex $(S=1,2$ and 3$)$ states in the self-attractive BEC $(g<0)$, under the action of the in-plane trapping potential (4) with $\lambda=0.1$ and $\zeta=1$. As indicated in the table, the critical values are calculated as per the full 3D-GPE and 2D approximations.

ticity $S$ was taken as

$$
\Phi(x, y, t=0)=\beta(x+i y)^{S} \exp \left[-\frac{\lambda}{2}\left(x^{2}+\frac{y^{2}}{\gamma^{2}}\right)\right],
$$

where $\beta$ is a constant determined by the normalization condition (27), and $\gamma$ is the parameter of the anisotropic deformation. Below, we set $\gamma=1.1$.

From the numerical results we conclude that the vortex solutions with $S=1$ are stable at $g \geqslant-3.1$. It is worth noting that the modulus of this value, which borders the stability region of the vortices with $S=1$, is much smaller than the modulus of the respective value at the collapse point, $g_{c}(S=1)=-8.1$, see Table I. In the stability region, perturbed vortex states show quasiperiodic oscillations, maintaining their integrity. These oscillations, featuring alternations of the ellipticity between the $x$ and $y$ axes (eccentricity oscillations [107]), are caused by the initially imposed anisotropy in Eq. (44). A typical example is shown in Fig. 8(a), where we plot a stable vortex profile with $S=1$ and $g=-1$ obtained at $t=300$ after nine cycles of the quasi-periodic eccentricity oscillations. These results are in agreement with those previously published for 2D NPSE [74] and also for cubic 2D NLSE [103-106].

In the instability region, i.e., at $g<-3.1$, the evolution leads to fission of the vortex ring in two fragments that rotate around the center and recombine (fuse) again. Near the instability border, $g=-3.1$, 

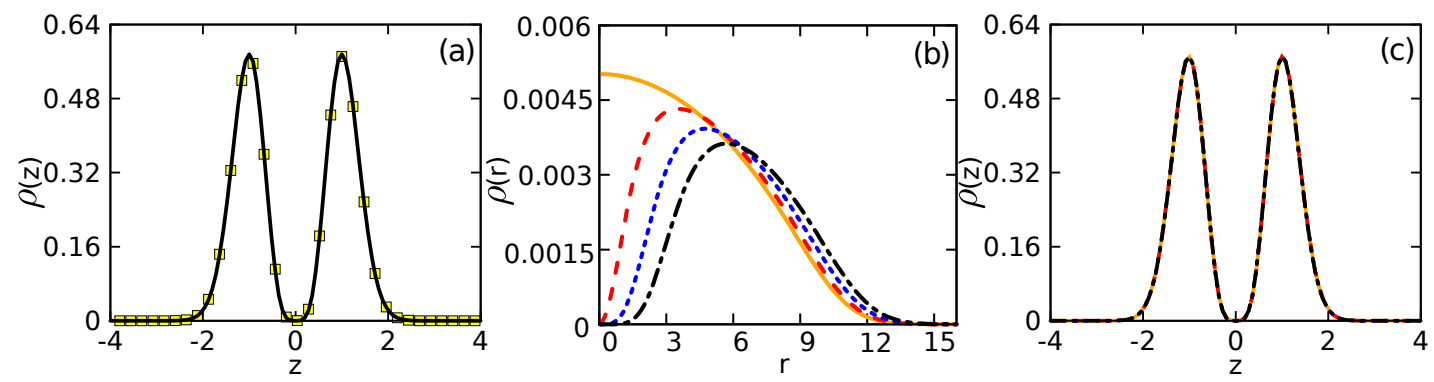

FIG. 7. (a) Lumped axial density profiles $\rho(z)$ for the repulsive condensate, with $\lambda=0.1, g=1$, and vorticity $S=2$, produced by the full 3D GPE and 2D NPSE (yellow squares and black solid lines, respectively. (b) Radial density profiles, $\rho(r)$, for the repulsive condensate with $g=50$, under the action of the in-plane trapping potential (4) with $\lambda=0.1$ and $\zeta=1$, as produced by the 2D NPSE with normalization (27), for different values of the vorticity: $S=0,1,2$, and 3 . They are depicted, respectively, by the orange solid, red dashed, blue dotted, and black dashed-dotted lines. (c) Axial density profiles, $\rho(z)$, for the same states as in panel (b). All the profiles are practically overlapping.
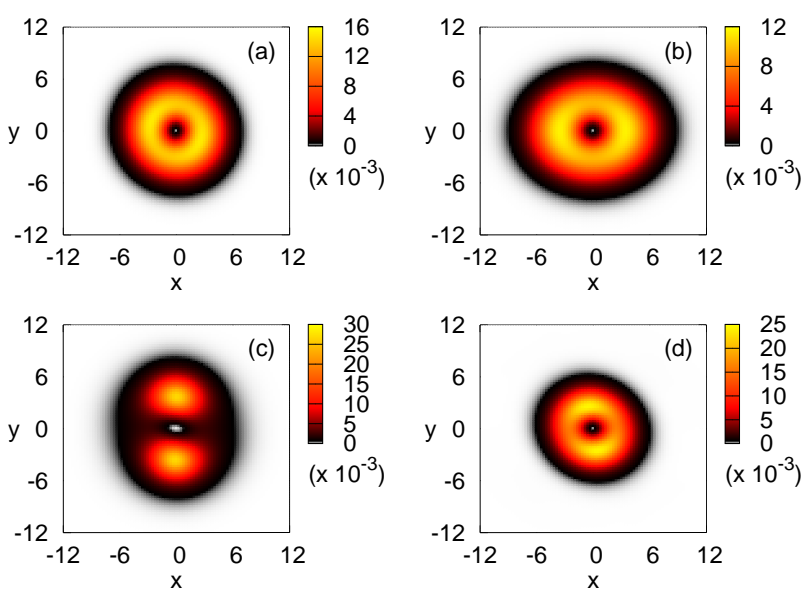

FIG. 8. Density profiles $|\Phi(x, y, t)|^{2}$ display the evolution of the vortex states, with $S=1$, which were initially subjected to the elliptic deformation, as per Eq. (44) with $\gamma=1.1$ and $\lambda=0.1$. The results were produced by simulations of Eq. (29) with the in-plane potential (4) and parameters $\zeta=1, \lambda=0.1$. (a): A snapshot, at $t=300$, of a stable vortex profile, for $g=$ -1 . Panels (b), (c) and (d) display snapshots, at $t=0,60$, and 140 , respectively, of the periodically splitting and recombining vortex profile, for $g=-3.3$.

fission-fusion cycles repeat quasi-periodically. For example, at $g=-3.3$ the period of this dynamical regime is $\tau_{\mathrm{ff}} \simeq 140$. These results are exhibited by means of snapshots in Figs. 8(b-d). For the same case, numerical data demonstrate that time necessary for the two fragments to make a complete rotation around the origin is $\tau_{\mathrm{r}} \simeq 80$. Thus, the fission-fusion cycles and rotation are not strictly synchronized, the ratio of the respective periods being

$$
\tau_{\mathrm{ff}} / \tau_{\mathrm{r}} \simeq 1.75
$$

In the regime of strong attractive self-interaction, the oscillation frequency increases with the increase of $|g|$, eventually leading to the onset of the collapse. For in-
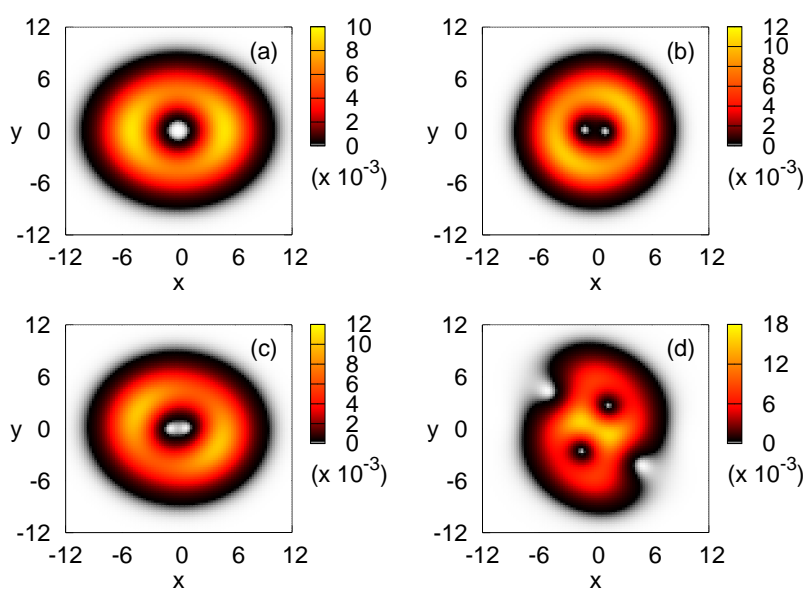

FIG. 9. The same as in Fig. 8, but for the vortex with $S=2$. Panels (a), (b) and (c) are snapshots of the solution's profile in the quasi-periodic fission-fusion regime, taken at $t=0$, and 50, and 100, respectively, for $g=-0.2$. (d) A snapshot of a permanently split (and gradually separating) rotating two-vortex pair, taken at $t=300$ for $g=-2$.

stance, at $g=-5$ and $S=1$, the evolution of the initial profile (44) ends up with the collapse at $t \simeq 31$.

For vortex states with $S=2$ no stability region was found, similar to what was reported earlier in the case of the 2D GPE with the cubic self-attraction and HO trapping potential $[103,106]$. In the case of weak attraction, the evolution of input (44) exhibits fission of the double vortex into a coupled pair of unitary vortices with two separated pivots. Then the pair fuses into a single double vortex, and the fission-fusion cycles for the vortices recur quasi-periodically. Simultaneously, the configuration features persistent rotation. This dynamical regime for the double vortex is illustrated, in Figs. 9(a-c), for $g=-0.2$ by dint of snapshots taken at $t=0,50,100$. In this case, the fission-fusion period is $\tau_{\mathrm{ff}} \simeq 31$. It is worthy to note that the respective oscillatory motion of pivots of the two unitary vortices proceeds along the $x$ - 

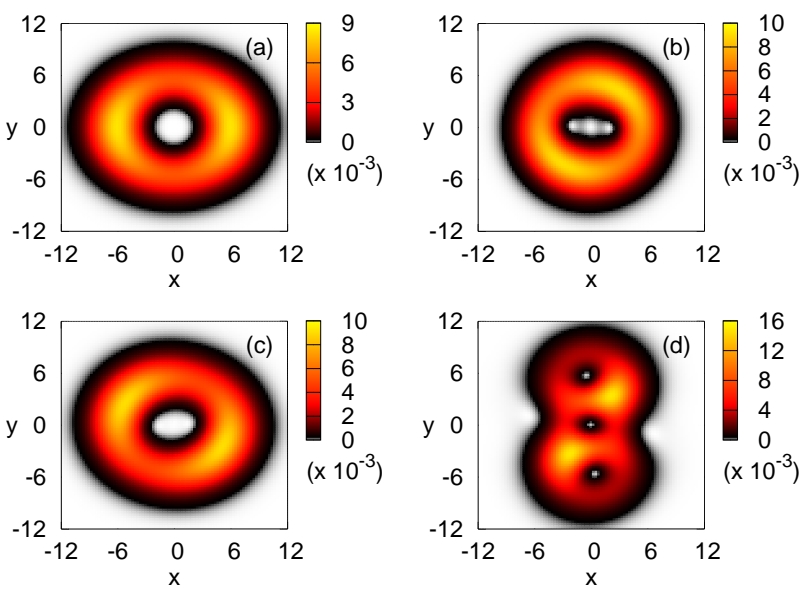

FIG. 10. The same as in Fig. 9, but for $S=3$.

axis, keeping $y=0$. Simultaneously, the position of the maximum local intensity of the solution rotates with a period of $\tau_{r} \simeq 62$. Thus, in this cases, the ratio of the periods is

$$
\tau_{\mathrm{ff}} / \tau_{\mathrm{r}} \simeq 0.5,
$$

quite different from the above value given by Eq. (45).

On the other hand, in the case of strong self-attraction, the double vortex permanently splits in a pair of gradually separating unitary vortices, which rotate around the center, unlike the dynamical scenario outlined above for the case of weak self-attraction. In spite of the difference, for the pair of separating unitary vortices the rotation period is found to be the same as observed for the rotation of the maximum amplitude in case of the weak self-attraction. The dynamics of the permanently split double vortex is displayed in Fig. 9(d), where local density $|\Phi(x, y, t)|^{2}$ is plotted in the $(x, y)$ plane for $g=-2$ at $t=300$.

The analysis was also developed to triple vortex states, with $S=3$, producing results similar to those reported above for $S=2$. The triplets are unstable against fission into a rotating set of three unitary vortices, whose pivots are aligned in the radial direction. For small values of $|g|$, such as $g=-0.2$, the secondary eddies temporarily fuse back into a single triple vortex, thus initiating a quasi-periodic sequence of fission-fusion cycles, as shown in Figs. 10(a-c). The cycles resemble those demonstrated above for vortices with $S=2$ in the case of the weak self-attraction with $S=2$, with the same values of the fission-fusion and overall-rotation periods, see Eq. (46). On the other hand, permanent splitting of the initial triple vortex in a rotating set of unitary vortices, gradually separating in the radial direction, takes place at larger values of $|g|$. An example of the set of three radially separating eddies is plotted in Fig. 10 (d) for $g=-2$ at $t=300$. In such a configuration, the separation between them values which are essentially larger than the maximum distance observed in the fission-fusion regime. The rotary motion of the vortex set and its period are similar to those demonstrated in the same regime for $S=2$ in Fig. 9(d).

Finally, to elucidate the dynamical scenarios of the instability development outlined above for the vortex states with $S=1,2$, and 3, in Fig. 11 we plot the evolution of ratio $\left\langle r^{2}\right\rangle /\left\langle x^{2}\right\rangle$ of the spatially averaged $2 \mathrm{D}$ radial variable, $r^{2}=x^{2}+y^{2}$, and the squared coordinate,

$$
\left\langle r^{2}, x^{2}\right\rangle \equiv \iint\left(r^{2}, x^{2}\right)|\phi(x, y, t)|^{2} d x d y,
$$

for $S=1,2$ and 3 (the definition of the average does not include a normalization factor, as it cancels in ratio $\left.\left\langle r^{2}\right\rangle /\left\langle x^{2}\right\rangle\right)$. The computation was performed using numerical solutions of the effective radial equation (41) in 2D Cartesian coordinates.

In Fig. 11(a) one observes quasi-periodic oscillations of $\left\langle r^{2}\right\rangle /\left\langle x^{2}\right\rangle$ in an unstable state with $S=1$ and $g=$ -3.3 , as a result of the superposition of the recurring fission-fusion cycles and overall rotation with different periods, see Eq. (45). Note that maxima of the ratio correspond to minima of $\left\langle x^{2}\right\rangle$, when the rotating unitary vortex is crossing axis $x=0$. On the other hand, in Figs. 11(b) and (c) unstable vortices with $S \geq 2$ and small $|g|$ demonstrate practically harmonic oscillations of the same ratio, $\left\langle R^{2}\right\rangle /\left\langle x^{2}\right\rangle$, which is explained by the fact that in this case the periods of the fission-fusion cycle and rotation are commensurable, see Eq. (46).

Finally, as shown above, unstable double and triple vortices, with $S=2$ and 3, at large $|g|$ irreversibly split into sets of two or three unitary vortices displaced along the radial direction, keeping to separate in this direction. Accordingly, the respective curves in Figs. 11(b) and 11(c) exhibit quasi-harmonic oscillations with a growing amplitude. Eventually, the separation halts under the action of the HO trapping potential in Eq. (41).

\section{CONCLUSION}

The objective of this work is to produce additional results for the important problem of the reduction of the full 3D dynamics of BEC, loaded in an external potential, which imposes strong confinement in one direction $(z)$, to an effective $2 \mathrm{D}$ form. Here, this general problem is considered for the specific form of the confining potential, given by Eq. (3), which is a combination of the singular repulsive term $\zeta^{2} / z^{2}$ and usual HO (harmonic-oscillator) trap. The singular term splits the $3 \mathrm{D}$ condensate into a pair of parallel non-interacting "pancakes", which is an example of the "superselection" phenomenon. A physical realization of this configuration is proposed, in terms of a resonant optical field, whose frequency is subjected to appropriate modulation in direction $z$, perpendicular to the "pancakes". The reduction of the underlying 3D GPE (Gross-Pitaevskii equation) to the 2D NPSE (nonpolynomial Schrödinger 

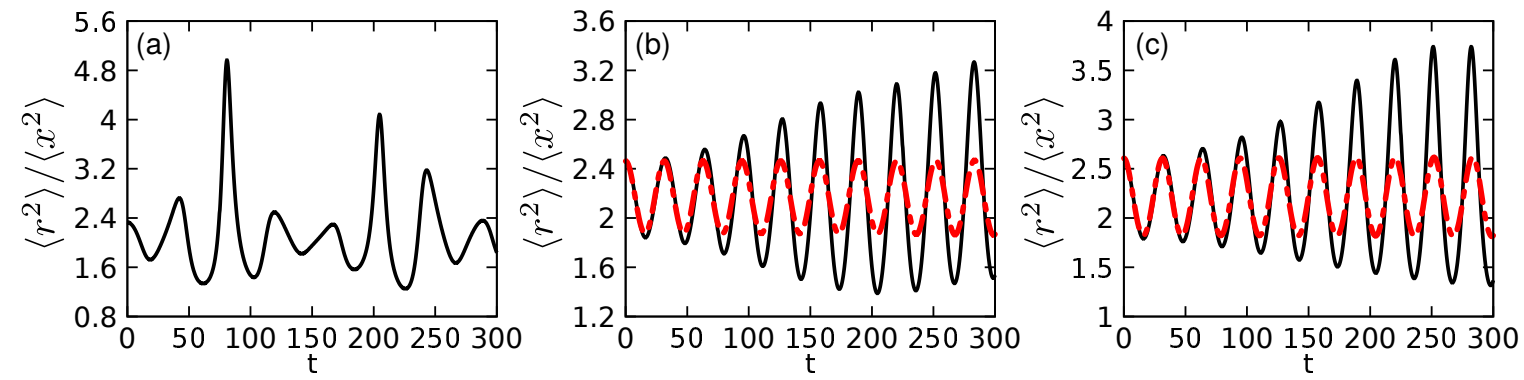

FIG. 11. The ratio of spatially averaged squared coordinates, $\left\langle r^{2}\right\rangle /\left\langle x^{2}\right\rangle$ [see Eq. (47)], versus time, illustrating the unstable evolution of initial vortex states with (a) $S=1$ and $g=-3.3$; (b) $S=2$ and $g=-0.2$ or -2 (the dashed red or solid black lines, respectively; (c) the same as in (b), but for $S=3$. The other parameters are $\lambda=0.1, \zeta=1$, and $\gamma=1.1$.

equation) is provided by the factorized ansatz, making use of the fact that the $z$-dependent potential admits an exact GS (ground-state) solution of the respective Schrödinger equation. The full spectrum of energy eigenvalues in this transverse potential is found in an exact form too. The potential demonstrates a quantum phase transition between the GS (ground state) of HO in the case of $\zeta^{2}=0$ and the "superselection" state at $\zeta^{2}>0$. The resulting two-dimensional NPSE (nonpolynomial Schrödinger equation), with the repulsive or attractive nonlinearity, produces GS and vortex-state solutions, and the threshold for the onset of the collapse, which are virtually identical to their counterparts obtained from the numerical solution of the underlying 3D GPE. On the other hand, the 2D NLSE with the usual cubic nonlinearity, as well as the TFA (ThomasFermi approximation), give rise to conspicuous discrepancies, in comparison to the full 3D solution. Thus, the results demonstrate high accuracy of the appropriately formulated spatial-dimensionality reduction. This method may be applied to other settings as well.

In particular, the existence of stable vorticity states with $S=1$ in the case of the self-attractive sign of the nonlinearity is demonstrated by direct simulations of the effective time-dependent 2D NPSE. On the other hand, all higher-order vortex states with $S=2$ and 3 are unstable. At relatively weak self-attraction strength, the instability triggers a quasi-periodic sequence of fissionfusion cycles, while stronger self-attraction irreversibly splits double and triple vortices in linearly arranged rotating sets of gradually separating unitary vortices.

As an extension of the present work, it may be interesting to consider more sophisticated patterns, such as necklace-shaped ones built of fundamental or vortex solitons, cf. necklace states found in various other models [108-111]. Another relevant extension is to consider dark solitons in the model with the self-repulsive nonlinearity, and without the axial confinement. Such modes can be created by means of the well-known phase-imprinting technique [112]. In particular, it may be interesting to consider moving dark solitons and collisions between them.

\section{ACKNOWLEDGMENTS}

The authors acknowledge financial support from the Brazilian agencies CNPq (\#425718/2018-2 \& \#306065/2019-3), CAPES, and FAPEG (PRONEM \#201710267000540 and PRONEX \#201710267000503). This work was performed under auspices of the Brazilian National Institute of Science and Technology (INCT) for Quantum Information (\#465469/2014-0). The work B.A.M. is supported, in part, by the Israel Science Foundation through Grant No. 1286/17, and by CAPES (Brazil) through program PRINT, grant No. 88887.364746/2019-00.
[1] L. Khaykovich, F. Schreck, G. Ferrari, T. Bourdel, J. Cubizolles, L. D. Carr, Y. Castin, and C. Salomon, Science (80-. ). 296, 1290 (2002).

[2] K. E. Strecker, G. B. Partridge, A. G. Truscott, and R. G. Hulet, Nature 417, 150 (2002).

[3] S. L. Cornish, S. T. Thompson, and C. E. Wieman, Phys. Rev. Lett. 96, 170401 (2006).

[4] A. L. Marchant, T. P. Billam, T. P. Wiles, M. M. H. Yu, S. A. Gardiner, and S. L. Cornish, Nat. Commun. 4, 1865 (2013).

[5] F. Kh. Abdullaev, A. Gammal, A. M. Kamchatnov, and L. Tomio, Int. J. Mod. Phys. B 19, 3415-3473 (2005).
[6] L. Salasnich, Opt. Quant. Electron. 49, 409 (2017).

[7] S. Burger, K. Bongs, S. Dettmer, W. Ertmer, K. Sengstock, A. Sanpera, G. V. Shlyapnikov, and M. Lewenstein, Phys. Rev. Lett. 83, 5198 (1999).

[8] C. Becker, S. Stellmer, P. Soltan-Panahi, S. Dörcher, M. Baumert, E.-M. Richter, J. Kronjäger, K. Bongs, and K. Sengstock, Nat. Phys. 4, 496 (2008).

[9] M. R. Matthews, B. P. Anderson, P. C. Haljan, D. S. Hall, C. E. Wieman, and E. A. Cornell, Phys. Rev. Lett. 83, 2498 (1999).

[10] T. W. Neely, E. C. Samson, A. S. Bradley, M. J. Davis, and B. P. Anderson, Phys. Rev. Lett. 104, 160401 (2010). 
[11] D. V. Freilich, D. M. Bianchi, A. M. Kaufman, T. K. Langin, and D. S. Hall, Science (80-. ). 329, 1182 (2010).

[12] J. A. Seman, E. A. L. Henn, M. Haque, R. F. Shiozaki, E. R. F. Ramos, M. Caracanhas, P. Castilho, C. Castelo Branco, P. E. S. Tavares, F. J. Poveda-Cuevas, G. Roati, K. M. F. Magalhães, and V. S. Bagnato, Phys. Rev. A 82, 033616 (2010).

[13] S. Middelkamp, P. J. Torres, P. G. Kevrekidis, D. J. Frantzeskakis, R. Carretero-González, P. Schmelcher, D. V. Freilich, and D. S. Hall, Phys. Rev. A 84, 011605(R) (2011).

[14] C. Ryu, M. F. Andersen, P. Cladé, V. Natarajan, K. Helmerson, and W. D. Phillips, Phys. Rev. Lett. 99, 260401 (2007).

[15] A. Ramanathan, K. C. Wright, S. R. Muniz, M. Zelan, W. T. Hill, C. J. Lobb, K. Helmerson, W. D. Phillips, and G. K. Campbell, Phys. Rev. Lett. 106, 130401 (2011).

[16] A. I. Yakimenko, Y. M. Bidasyuk, M. Weyrauch, Y. I. Kuriatnikov, and S. I. Vilchinskii, Phys. Rev. A 91, 033607 (2015).

[17] S. Wuester, T. E. Argue, and C. M. Savage, Phys. Rev. A 72, 043616 (2005).

[18] Y.-J. Lin, R. L. Compton, K. Jiménez, W. D. Phillips, J. V. Porto, and I. B. Spielman, Nat. Phys. 7, 531 (2011).

[19] Y.-J. Lin, K. Jiménez, and I. B. Spielman, Nature 471, 83 (2011).

[20] T. Kinoshita, T. Wenger, and D. S. Weiss, Nature 440, 900 (2006).

[21] J. Billy, V. Josse, Z. Zuo, A. Bernard, B. Hambrecht, P. Lugan, D. Clément, L. Sanchez-Palencia, P. Bouyer, and A. Aspect, Nature 453, 891 (2008).

[22] G. Roati, C. D. Errico, L. Fallani, M. Fattori, C. Fort, M. Zaccanti, G. Modugno, M. Modugno, and M. Inguscio, Nature 453, 895 (2008).

[23] E. G. Charalampidis, J. Cuevas-Maraver, D. J. Frantzeskakis, and P. G. Kevrekidis, Rom. Reports Phys. 70, 504 (2018).

[24] D. S. Petrov, Phys. Rev. Lett. 115, 155302 (2015).

[25] D. S. Petrov and G. E. Astrakharchik, Phys. Rev. Lett. 117, 100401 (2016).

[26] M. Schmitt, M. Wenzel, F. Böttcher, I. Ferrier-Barbut, and T. Pfau, Nature 539, 259 (2016).

[27] C. R. Cabrera, L. Tanzi, J. Sanz, B. Naylor, P. Thomas, P. Cheiney, and L. Tarruell, Science (80-. ). 359, 301 (2018).

[28] G. Semeghini, G. Ferioli, L. Masi, C. Mazzinghi, L. Wolswijk, F. Minardi, M. Modugno, G. Modugno, M. Inguscio, and M. Fattori, Phys. Rev. Lett. 120, 235301 (2018).

[29] C. DErrico, A. Burchianti, M. Prevedelli, L. Salasnich, F. Ancilotto, M. Modugno, F. Minardi, and C. Fort, Phys. Rev. Res. 1, 033155 (2019).

[30] Y. Li, Z. Chen, Z. Luo, C. Huang, H. Tan, W. Pang, and B. A. Malomed, Phys. Rev. A 98, 063602 (2018).

[31] M. N. Tengstrand, P. Stürmer, E. Ö. Karabulut, and S. M. Reimann, Phys. Rev. Lett. 123, 160405 (2019).

[32] Y. V. Kartashov, B. A. Malomed, and L. Torner, Phys. Rev. Lett. 122, 193902 (2019).

[33] I. Morera, G. E. Astrakharchik, A. Polls, and B. JuliaDiaz, Phys. Rev. Res. 2, 022008 (2020).

[34] F. Dalfovo, S. Giorgini, L. P. Pitaevskii, and S. Stringari, Rev. Mod. Phys. 71, 463 (1999).

[35] J. Weiner, V. S. Bagnato, S. Zilio, and P. S. Julienne, Rev. Mod. Phys. 71, 1 (1999).

[36] I. Bloch, J. Dalibard, and W. Zwerger, Rev. Mod. Phys. 80,885 (2008).
[37] V. A. Brazhnyi and V. V. Konotop, Mod. Phys. Lett. B 18, 627 (2004).

[38] F. Kh. Abdullaev, A. Gammal, A. M. Kamchatnov, and L. Tomio, Int. J. Mod. Phys. B 19, 3415 (2005).

[39] R. Gati and M. K. Oberthaler, J. Phys. B At. Mol. Opt. Phys. 40, R61 (2007).

[40] A. L. Fetter, Rev. Mod. Phys. 81, 647 (2009).

[41] D. J. Frantzeskakis, J. Phys. A Math. Theor. 43, 213001 (2010).

[42] T. Lahaye, C. Menotti, L. Santos, M. Lewenstein, and T. Pfau, Reports Prog. Phys. 72, 126401 (2009).

[43] S.-W. Song, L. Wen, C.-F. Liu, S.-C. Gou, and W.-M. Liu, Front. Phys. 8, 302 (2013).

[44] M. Ueda, Reports Prog. Phys. 77, 122401 (2014).

[45] H. Zhai, Reports Prog. Phys. 78, 026001 (2015).

[46] V. S. Bagnato, D. J. Frantzeskakis, P. G. Kevrekidis, B. A. Malomed, and D. Mihalache, Rom. Reports Phys. 67, 5 (2015).

[47] Y.-J. Lin and I. B. Spielman, J. Phys. B At. Mol. Opt. Phys. 49, 183001 (2016).

[48] Y. Zhang, M. E. Mossman, T. Busch, P. Engels, and C. Zhang, Front. Phys. 11, 118103 (2016).

[49] S. A. Moses, J. P. Covey, M. T. Miecnikowski, D. S. Jin, and J. Ye, Nat. Phys. 13, 13 (2017).

[50] L. Salasnich, Opt. Quantum Electron. 49, 409 (2017).

[51] H. Sakaguchi, Front. Phys. 14, 12301 (2019).

[52] D.-W. Zhang, Y.-Q. Zhu, Y. X. Zhao, H. Yan, and S.-L. Zhu, Adv. Phys. 67, 253 (2018).

[53] Y. V. Kartashov, G. E. Astrakharchik, B. A. Malomed, and L. Torner, Nat. Rev. Phys. 1, 185 (2019).

[54] V. Bagnato and D. Kleppner, Phys. Rev. A 44, 7439 (1991).

[55] B. Malomed, Condens. Matter 3, 15 (2018).

[56] A. Görlitz, J. M. Vogels, A. E. Leanhardt, C. Raman, T. L. Gustavson, J. R. Abo-Shaeer, A. P. Chikkatur, S. Gupta, S. Inouye, T. Rosenband, and W. Ketterle, Phys. Rev. Lett. 87, 130402 (2001).

[57] L. Salasnich, A. Parola, and L. Reatto, Phys. Rev. A 65, 043614 (2002).

[58] L. Salasnich, A. Parola, and L. Reatto, Phys. Rev. A 66, 043603 (2002).

[59] L. Salasnich, A. Cetoli, B. A. Malomed, and F. Toigo, Phys. Rev. A 75, 033622 (2007).

[60] A. Maluckov, L. Hadzievski, B. A. Malomed, and L. Salasnich, Phys. Rev. A 78, 013616 (2008).

[61] S. K. Adhikari and L. Salasnich, New J. Phys. 11, 023011 (2009).

[62] L. Salasnich, J. Phys. A Math. Theor. 42, 335205 (2009).

[63] Luis E. Young-S, L. Salasnich, and S. K. Adhikari, Phys. Rev. A 82, 053601 (2010).

[64] L. Salasnich and B. A. Malomed, J. Phys. B At. Mol. Opt. Phys. 45, 055302 (2012).

[65] L. Salasnich and B. A. Malomed, Phys. Rev. A 87, 063625 (2013).

[66] Luis E. Young-S, L. Salasnich, and B. A. Malomed, Phys. Rev. A 87, 043603 (2013).

[67] W. B. Cardoso, L. Salasnich, and B. A. Malomed, Eur. Phys. J. D 71, 112 (2017).

[68] W. B. Cardoso, L. Salasnich, and B. A. Malomed, Sci. Rep. 7, 16876 (2017).

[69] A. Pendse and A. Bhattacharyay, J. Phys. Condens. Matter 30, 455602 (2018).

[70] H. L. C. Couto, A. T. Avelar, and W. B. Cardoso, Ann. Phys. 530, 1700352 (2018). 
[71] M. C. P. dos Santos and W. B. Cardoso, Phys. Lett. A 383, 1435 (2019).

[72] F. Gerbier, EPL 66, 771 (2004).

[73] A. Muñoz Mateo and V. Delgado, Phys. Rev. A 77, 013617 (2008).

[74] L. Salasnich and B. A. Malomed, Phys. Rev. A 79, 053620 (2009).

[75] G. Gligorić, A. Maluckov, M. Stepić, L. Hadzievski, and B. A. Malomed, Phys. Rev. A 81, 013633 (2010).

[76] M. Edwards, M. Krygier, H. Seddiqi, B. Benton, and C. W. Clark, Phys. Rev. E 86, 056710 (2012).

[77] L. Salasnich, W. B. Cardoso, and B. A. Malomed, Phys. Rev. A 90, 033629 (2014).

[78] R. K. Kumar, L. Tomio, B. A. Malomed, and A. Gammal, Phys. Rev. A 96, 063624 (2017).

[79] W. D. Phillips, Rev. Mod. Phys. 70, 721 (1998).

[80] J. Denschlag and J. Schmiedmayer, Europhys. Lett. 38, 405 (1997).

[81] J. Denschlag, G. Umshaus, and J. Schmiedmayer, Phys. Rev. Lett. 81, 737 (1998).

[82] H. Sakaguchi and B. A. Malomed, Phys. Rev. A 83, 013607 (2011).

[83] H. Sakaguchi and B. A. Malomed, Phys. Rev. A 84, 033616 (2011).

[84] G. E. Astrakharchik and B. A. Malomed, Phys. Rev. A 92, 043632 (2015)

[85] E. Shamriz, Z. Chen, B. A. Malomed, and H. Sakaguchi, Condens. Matter 5, 20 (2020).

[86] L. D. Landau and E. M. Lifshitz, Quantum Mechanics (Elsevier, 1977).

[87] L. P. Pitaevskii and S. Stringari, Bose-Einstein Condensation (Clarendon Press, 2003).

[88] C. J. Pethick and H. Smith, Bose-Einstein Condensation in Dilute Gases (Cambridge University Press, Cambridge, 2008).

[89] J. A. Devlin and M. R. Tarbutt, New J. Phys. 18, 123017 (2016).

[90] K. N. Jarvis, J. A. Devlin, T. E. Wall, B. E. Sauer, and M. R. Tarbutt, Phys. Rev. Lett. 120, 083201 (2018).

[91] Y. Li, J. Liu, W. Pang, and B. A. Malomed, Phys. Rev. A 88,053630 (2013).
[92] P. M. Drljača, F. Vincent, P.-A. Besse, and R. S. Popović, Sensors and Actuators A 97-98, 10 (2002).

[93] B. A. Malomed, Spontaneous Symmetry Breaking, SelfTrapping, and Josephson Oscillations (Springer Berlin Heidelberg, Berlin, Heidelberg, 2013).

[94] M. Ávila-Aoki, C. Cisneros, R. P. Martínez-y-Romero, H. N. Núñez-Yéepez, and A. L. Salas-Brito, Phys. Lett. A 373, 418 (2009).

[95] D. Viennot and L. Aubourg, Phys. Lett. A 380, 678 (2016).

[96] H.-Y. Xu, G.-L. Wang, L. Huang, and Y. C. Lai, Phys. Rev. Lett. 120, 124101 (2018).

[97] J. Yang, Nonlinear Waves in Integrable and Nonintegrable Systems (Society for Industrial and Applied Mathematics, 2010).

[98] P. Muruganandam and S. K. Adhikari, Comput. Phys. Commun. 180, 1888 (2009).

[99] L. E. Young-S., P. Muruganandam, S. K. Adhikari, V. Lončar, D. Vudragović, and A. Balaž, Comput. Phys. Commun. 220, 503 (2017).

[100] H. Sakaguchi and B. A. Malomed, Phys. Rev. A 81, 013624 (2010).

[101] G. Fibich, The Nonlinear Schrödinger Equation (Springer International Publishing, Cham, 2015).

[102] J. Qin, G. Dong, and B. A. Malomed, Phys. Rev. A 94, 053611 (2016).

[103] D. Mihalache, D. Mazilu, B. A. Malomed, and F. Lederer, Phys. Rev. A 73, 043615 (2006).

[104] T. J. Alexander and L. Bergé, Phys. Rev. E 65, 026611 (2002).

[105] H. Saito and M. Ueda, Phys. Rev. Lett. 89, 190402 (2002).

[106] B. A. Malomed, Physica D 399, 108 (2019).

[107] P. L. Christiansen, N. Gronbech-Jensen, P. S. Lomdahl, and B. A. Malomed, Phys. Scr. 55, 131 (1997).

[108] M. Soljačić, S. Sears, and M. Segev, Phys. Rev. Lett. 81, 4851 (1998).

[109] A. S. Desyatnikov and Y. S. Kivshar, Phys. Rev. Lett. 87, 033901 (2001).

[110] Y. V. Kartashov, L.-C. Crasovan, D. Mihalache, and L. Torner, Phys. Rev. Lett. 89, 273902 (2002).

[111] T. D. Grow, A. A. Ishaaya, L. T. Vuong, and A. L. Gaeta, Phys. Rev. Lett. 99, 133902 (2007).

[112] P. G. Kevrekidis, R. Carretero-González, D. J. Frantzeskakis, and I. G. Kevrekidis, Mod. Phys. B 18,1481 (2004). 